\title{
Vitiligo and disorders of the retinal pigment epithelium
}

\author{
DANIEL M. ALBERT, MICHAEL D. WAGONER, RONALD C. PRUETT, \\ JAMES J. NORDLUND, AND AARON B. LERNER
}

From the Department of Ophthalmology, Harvard Medical School, Massachusetts Eye and Ear Infirmary, Boston, Massachusetts, USA

SUMmARY The association of vitiligo with inflammation of the uveal tract is well established. The relationship between vitiligo and hypopigmentation and/or degeneration of the retinal pigment epithelium (RPE) not secondary to ocular inflammation has not been adequately investigated. Sixty (27\%) of 223 consecutive patients with vitiligo were found to have some evidence of RPE hypopigmentation ranging from mild, focal areas of involvement in most cases to extensive RPE degeneration with a retinitis pigmentosa-like syndrome in one patient. Fifteen $(25 \%)$ patients complained of night blindness. Only $6(4 \%)$ of 148 patients in a control group had similar funduscopic findings $(p<0 \cdot 001)$. None of these patients were symptomatic. There have been isolated reports of vitiligo occurring with tapetoretinal degeneration. We report 2 patients with both vitiligo and retinitis pigmentosa.

Vitiligo is defined as an 'idiopathic, probably autoimmune condition characterized by destruction of the melanocytes in small or large circumscribed areas of the skin, resulting in patches of depigmentation, often having a hyperpigmented border or often enlarging slowly'. ${ }^{1}$ It affects approximately $0.38 \%$ to $1.0 \%$ of the population. ${ }^{23}$

Depigmentation of the lids and poliosis of the brows and eyelashes are commonly seen in vitiligo. The association of vitiligo with inflammation of the uveal tract has long been recognised ${ }^{4-7}$ and is at present the subject of renewed interest. ${ }^{8-11}$ There have also been isolated reports of vitiligo occurring with tapetoretinal degenerations. ${ }^{12-17}$ In the present report we consider the possible relationship between vitiligo and disorders of the retinal pigment epithelium (RPE).

\section{Materials and methods}

The common finding of vitiligo and hypopigmentation and/or degeneration of the RPE was observed in patients drawn from 2 sources.

Two hundred and twenty-three patients with previously documented cutaneous vitiligo were examined for ocular abnormalities. These patients were seen consecutively at the Pigment Clinic of the Yale New Haven Hospital between July 1977 and February 1982 and did not represent patients with preselected ocular Correspondence to Daniel M. Albert, MD, Massachusetts Eye and Ear Infirmary, 243 Charles Street, Boston, MA 02114, USA. disease. A complete ophthalmological, dermatological, and family history was obtained. Each patient received a standard eye examination with careful recording of RPE hypopigmentation not related to previous or active chorioretinitis or senile macular degeneration. Eleven patients with RPE hypopigmentation were referred to the Electroretinography (ERG) Service at the Massachusetts Eye and Ear Infirmary (MEEI) for electroretinographic and psychological testing by methods previously described ${ }^{18}$ to exclude widespread retinal malfunction.

The age range of the vitiligo patients was 2-82 years, with an average age of 43 years; 143 were female; 83 were male; there were 202 Caucasians and 21 blacks. The duration of the vitiligo ranged from 5 months to 66 years, with an average of 13 years. Most of the patients were on psoralen therapy for their disease.

One hundred and forty-eight patients with psoriasis undergoing psoralen and ultraviolet light (PUVA) therapy were examined in an identical manner. This was an ideal control population because of a similar age range (5-77 years), average age (47 years), racial distribution (140 Caucasian, 6 black), and medication regimen (psoralen therapy) as a study group. Because of the nature of the skin changes in the vitiligo patients it was not possible to carry out a double-masked study.

We examined 2 patients in whom both vitiligo and retinitis pigmentosa (RP) were present. 


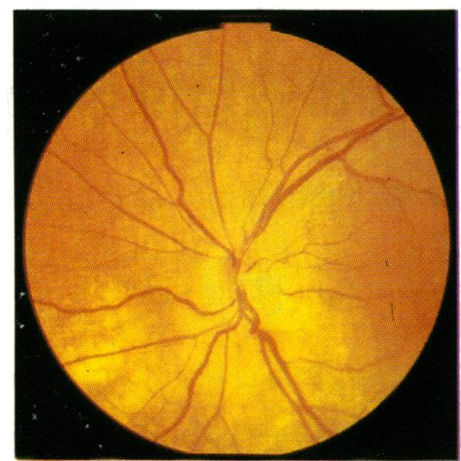

Fig. 1 Diffuse RPE atrophy with prominent choroidal pattern.

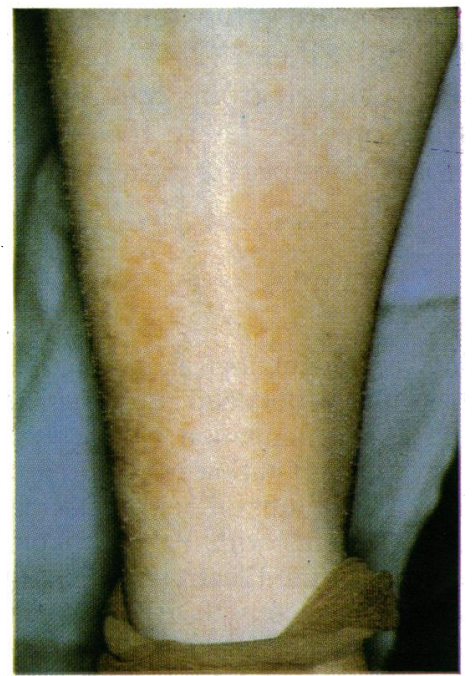

Fig. 4 Only small areas of lower leg pigment remain in this patient with total body vitiligo.

\section{Results}

The results are summarised in Table 1 . There was a statistically increased incidence of RPE hypopigmentation or atrophy in the vitiligo patients $(p<0.001)$. Forty-five of these patients were asymptomatic. Most of the patients had only focal areas of RPE hypopigmentation or a prominent choroidal pattern (Fig. 1). In some cases sectoral or geographic areas of RPE atrophy were present (Figs. 2 and 3). Eight of these patients had normal full field ERG studies, excluding extensive RPE degeneration.

Fifteen patients complained of nyctolopia. Three of these had full field ERG studies. Two were normal. One did have a significant decrease in both visual acuity as well as night blindness, which could be
Table 1 Vitiligo and RPE atrophy

\begin{tabular}{lcc}
\hline & Vitiligo & Psoriasis \\
\hline Number & 223 & 148 \\
RPE atrophy: & $60(27 \%)$ & $6(4 \%)$ \\
$\begin{array}{c}\text { asymptomatic } \\
\text { (normal ERG 8. } \\
\text { abnormal ERG 0) }\end{array}$ & 45 & 6 \\
$\begin{array}{c}\text { symptomatic } \\
\text { (normal ERG 2, }\end{array}$ & 15 & \\
$\quad$ abnormal ERG 1) & & 0 \\
\hline
\end{tabular}

accounted for on the basis of extensive RPE-photoreceptor degeneration. This patient was a 65 -year-old female of Albanian origin with no family history of retinitis pigmentosa or vitiligo. She denied having received any medications which could produce a pigmentary retinopathy. She developed vitiligo of the arms and hands at the age of 29 . This later progressed to complete body involvement with only limited areas of pigment remaining on the leg (Fig. 4). At the age of 44 she noticed a painless, progressive loss of night vision. ERG studies performed in 1965 and 1969 showed a progressive rod and cone degeneration. When we saw her in August 1981, the following pertinent findings were present: best corrected visual 


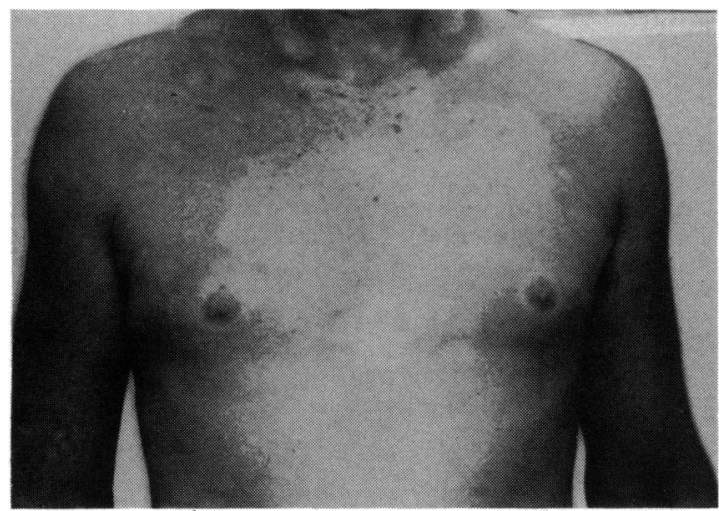

Fig. 6 Extensive vitiligo of the anterior chest.

acuity 20/400 OU. There was waxy pallor of each optic disc, retinal arteriolar narrowing, and fine areas of RPE atrophy throughout the periphery, with occasional whit deposits at the level of the RPE (Fig. 5). There was no vitreous cellular reaction or other evidence of intraocular inflammation. ERG testing showed no detectable rod or cone responses to scotopically matched blue and red light. With white light there was a small signal that was reduced in amplitude. The cone response to flicker at 30 cycles per second was undetectable. Dark adaptation studies with an $11^{\circ}$ white test flashlight showed a final threshold raised 3 to $3.5 \log$ units above normal. A diagnosis of retinitis pigmentosa sine pigmento or inverse retinitis pigmentosa could not be excluded.

We have seen 2 patients in whom vitiligo and retinitis pigmentosa were both present. The first was a 37-year-old male who first noted the onset of difficulty seeing in the dark at age 8 . At approximately the same time he developed vitiligo on both legs. This later involved his hands, arms, face, and trunk (Fig. 6). At the age of 25 the diagnosis of retinitis pigmen-

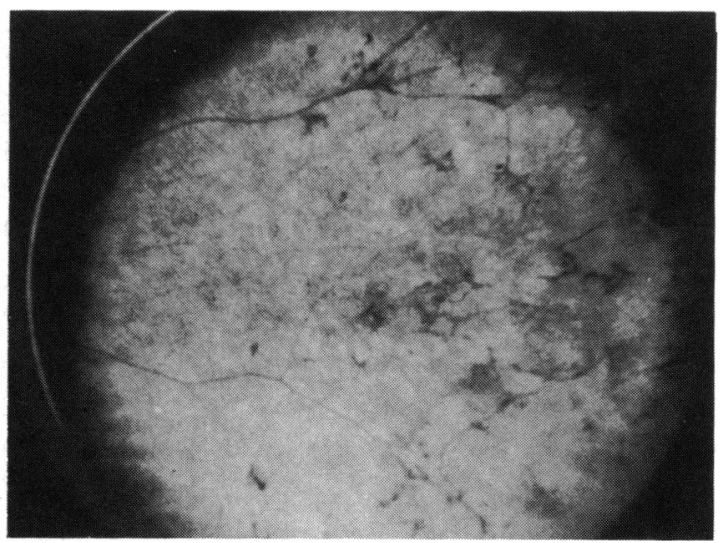

Fig. 7 Classic 'bone-spicules' of retinitis pigmentosa.

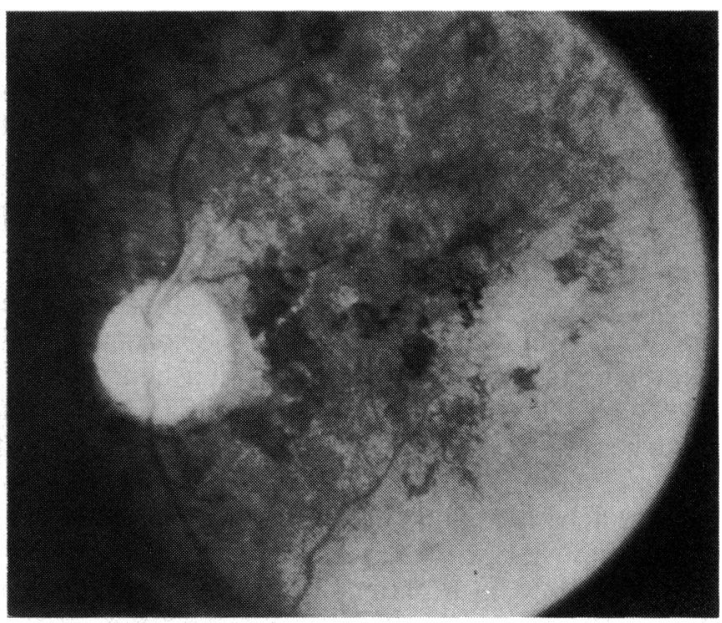

Fig. 8 Classic 'bone spicules' of retinitis pigmentosa.

tosa was made during a routine examination. His paternal grandfather had retinitis pigmentosa, but neither of his parents was known to be affected. He has 2 older brothers, one of whom has retinitis pigmentosa. His affected brother has a daughter with vitiligo but not retinitis pigmentosa. His ophthalmological examination revealed the following pertinent findings: best corrected visual acuity OD $20 / 50$, OS 20/100. The anterior vitreous contained pigment dispersion. There was waxy pallor of the optic disc, retinal arteriolar narrowing, and classic bone spicule formation extending from the arcades anterior to the equator (Fig. 7). Visual fields to confrontation were constricted to approximately $5^{\circ} \mathrm{OU}$. ERG responses were undetectable $\mathrm{OU}$.

The second patient was a 55-year-old black female with a 35-year history of retinitis pigmentosa, hearing loss, and vitiligo involving her mouth, scalp, and skin. The onset of her vitiligo occurred in her late teens. She had 3 sisters with retinitis pigmentosa, and hearing loss but no vitiligo. Her best corrected visual acuity was OD hand motions only, OS counting fingers at 5 feet $(1.5 \mathrm{~m})$. There were moderately advanced posterior subcapsular cataracts and pigment dispersion in the anterior vitreous. The optic disc was atrophic, the retinal arterioles were markedly narrowed, and there was very heavy bone spicule formation (Fig. 8). The visual fields were constricted to less than $5^{\circ} \mathrm{OU}$ to confrontation. ERG responses were undetectable.

\section{Discussion}

The hallmark of vitiligo is the destruction of melanocytes of the skin. Systemic vitiligo may involve the melanin-containing cells of the leptomeninges, inner 
ear, and eye, as well as cutaneous melanocytes. ${ }^{3-71119}$ In addition to depigmentation of the lids and poliosis of the eyelashes and brow, ${ }^{4}$ ocular involvement with vitiligo may include inflammation of the uveal tract, $^{\text {s-11 }}$ chorioretinal pigmentary disturbance, ${ }^{11}$ (Wagoner et al., unpublished), retinal pigment epithelium hypopigmentation and/or degeneration, ${ }^{19}$ or some combination of these.

The association of vitiligo with uveal inflammation in the Vogt-Koyanagi-Harada syndrome and sympathetic ophthalmia is well established..$^{5-7} 102021$ We have demonstrated an increased incidence of both active uveitis (Wagoner et al., unpublished), and healed chorioretinitis ${ }^{11}$ (Wagoner et al., unpublished), in vitiligo patients.

One case of severe bilateral RPE degeneration has been reported in a patient with vitiligo ${ }^{21}$ in whom there was no evidence of ocular inflammation. To the best of our knowledge, however, there has never been a systematic study of a large number of vitiligo patients to determine if they have an increased incidence of RPE hypopigmentation and/or degeneration not related to inflammation. We have demonstrated a significantly increased prevalence of nonspecific RPE hypopigmentation compared with a control population. Although $15(25 \%)$ of 60 patients complained of nyctalopia, only one of these patients who had ERG studies had decreased visual acuity and abnormal ERG and dark-adaptation studies which could be attributed to RPE-photoreceptor atrophy.

The relationship between vitiligo and retinitis pigmentosa is more difficult to assess. While this association has been reported infrequently, ${ }^{12-17}$ it is doubtful how thoroughly it has been investigated. We have described 2 patients in whom both disorders were simultaneously present. One of them had a family history of vitiligo. In addition we described one patient who may have retinitis pigmentosa sine pigmento or inverse retinitis pigmentosa. Of some interest was our finding that $4(1.7 \%)$ of the 223 vitiligo patients had a positive family history of retinitis pigmentosa. While this is not amenable to statistical analysis, it is suggestive of a possible association of the 2 disorders. We are currently undertaking a multicentre investigation to determine if there is an increased prevalence of vitiligo in patients with retinitis pigmentosa.

In summary, we have presented evidence suggesting an association between vitiligo and RPE hypopigmentation and/or degeneration. In most cases mild asymptomatic hypopigmentation of the RPE is seen associated with a generalised systemic pigmentary disturbance, but on rare occasions there may be sufficient RPE degeneration to produce a retinitis pigmentosa-like syndrome. Furthermore, it is our impression that there may be more than a random correlation between vitiligo and retinitis pigmentosa. More extensive investigation is under way to investigate this association.

We thank Dr Claude L. Cowans, chief of ophthalmology in the Department of Medicine at Howard University, and Dr Pearl Grimes, at the Department of Dermatology at Howard University. for referring the second vitiligo-retinitis pigmentosa patient to us. We also thank Dr Eliot Berson for performing the electroretinography studies and for his invaluable assistance in the preparation of this manuscript.

This project was supported in part by grant number NIAMDD 25252 entitled 'Ocular changes in vitiligo'.

\section{References}

1 Friel JP, ed. Dorlands Illustrated Medical Dictionary. 25th ed. Philadelphia: Saunders, 1974.

2 Horwitz J, Brodthagen H, Schwartz M. Prevalence of vitiligo. Arch Dermatol 1977; 113: 47-52.

3 Lerner $\mathrm{AB}$, Nordlund JJ. Vitiligo: What is it? Is it important? JAMA 1978; 239: 1183-7.

4 Wexler D. Ocular depigmentation accompanying generalized vitiligo. Arch Ophthalmol 1928; 57: 393-6.

5 Nettleship E. A case of sympathetic ophthalmitis with whitening of the eyelashes. Trans Ophthalmol Soc UK 1883-4; 4: 83-4.

6 Ikui H. Furuyoshi Y, Nakamizo K. Histopathological studies on cutaneous lesions in sympathetic ophthalmia and VogtKoyanagi-Harada syndrome. Nippon Ganka Gakkai Zasshi 1961; 65: $1057-9$.

7 Perry HD, Font RL. Clinical and histopathologic observations in severe Vogt-Koyanagi-Harada syndrome. Am JOphthalmol 1977; 83: $242-54$.

8 Ryan SJ. Maumenee AE. Birdshot retinochoroidopathy. Am J Ophthalmol 1979; 86: 1145--58.

9 Gass JDM. Vitiliginous chorioretinitis. Arch Ophthalmol 1981; 99: $1178-87$.

10 Nordlund JJ, Todes-Taylor N, Albert DM, Wagoner MD, Lerner AB. The prevalence of vitiligo and poliosis in patients with uveitis. J Am Acad Dermatol 1981; 4: 528-36.

11 Albert DM. Nordlund JJ, Lerner AB. Ocular abnormalities occurring with vitiligo. Ophthalmology 1979; 86: 1145-58.

12 Gordon DM. Retinitis pigmentosa 'sine pigmenti' associated with vitiligo of the skin. Arch Ophthalmol 1953; 50: 372-4.

13 Merz M, Szigielski M, Langucki J. Unilateral sectorial pigmentary degeneration and vitiligo. Ophthalmologica 1969; 157: 357-61.

14 Casale AM. Alezzandrini AA. Vitiligo y poliosis unilateral con retinitis pigmentaria y hipoacusia. Arch Argent Dermatol 1959; 9: 449-56.

15 Alezzandrini AA. Manifestation unilaterale de dégénérescence tapeto-retinienne de vitiligo, de poliose, de cheveux blancs et d'hypoacousie. Ophthalmologica 1964; 147: 409-19.

16 Haye MC. MMe Guyot-Sionnest, Coulon MG. Association de rétinite pigmentaire et de vitiligo. Bull Soc Ophtalmol Fr 1973; 73: 1155-8.

17 Makishita H, Inove K, Kagaya H, Yanagisawa N, Tsukagoshi H. Familial juvenile parkinsonism with retinitis pigmentosa and vitiligo. Rinsho Shinkeigaku 1978; 18: 143-8.

18 Berson EL, Gouras P, Gunkel RD. Rod responses in retinitis pigmentosa, dominantly inherited. Arch Ophthalmol 1968; 80: 58-67.

19 Kimura RS. Distribution, structure, and function of dark cells in the vestibular labyrinth. Ann Otol Rhinol Laryngol 1969; 78: 542-72.

20 Rosenbaum J, Bunke A, Cooperman E, Gombos GM. Bilateral retinal pigment epithelium changes associated with periorbital vitiligo and seizure disorders. Ann Ophthalmol 1979; 11: 1191-3.

21 Duke-Elder S. System of Ophthalmology. St Louis: Mosby, 1966: 9: 373 . 